\title{
EP-174
}

\section{Pancreatic solid harmatoma mimicking neuroendocrine tumor: Case report}

\author{
Jae Do YANG, Mi Rin LEE, Sung Woo AHN, Hee Chul YU*
}

Department of Surgery, Jeonbuk National University Hospital, Jeonju, Korea

Introduction: Pancreatic solid hamartoma is an extremely rare entity that shows components that are present in the normal pancreas with distorted architecture and are considered as malformative lesions. Preoperative diagnosis is difficult because the clinicopathological features of pancreatic solid hamartoma have not yet been fully clarified.

Methods: Herein, we report extremely rare case of pancreatic solid harmatoma mimicking neuroendocrine tumor.

Results: A 68-year-old male presented to our hospital for the evaluation of an incidentally detected pancreatic mass. Serum tumor marker levels were within normal limits. On delayed-phase computed tomography (CT), a homogeneous enhancing hypervascular mass measuring $1.8 \mathrm{~cm}$ was detected in the pancreatic head. On fluorodeoxyglucose-positron emission tomography (FDG-PET)/CT, the mass showed no significant FDG uptake. As the radiologic impression was that of a neuroendocrine tumor, the patient underwent pylorus-preserving pancreatoduodectomy. Pathologic findings show that microscopically, the tumor exhibited typical features of pancreatic solid hamartoma with no evidence of neuroendocrine differentiation in the cells of the lesion.

Conclusions: In conclusion, pancreatic hamartoma may be detected incidentally, without the patient presenting with any signs or symptoms of disease. Clinical symptoms are dependent on tumor size and location, and the diagnosis of this disease primarily depends on imaging techniques, including computed tomography, MRI and endoscopic ultrasound. However, pancreatic hamartoma is difficult to differentiate from other benign pancreatic lesions. For symptomatic patients or those with an indefinite diagnosis, a complete surgical excision is recommended. 